\title{
Pouvoir et consentement : quelques réflexions impressionnistes.
}

Ma principale (et brève) rencontre avec l'analyse stratégique fut la lecture attentive de "L'acteur et le système ", il y a très exactement vingt ans, pour l'examen du cours d'Olgierd Kuty. Je n'ai jamais regretté le temps consacré à cet ouvrage que j'avais considéré comme un remarquable " antidote » à ce que ma formation marxiste avait parfois laissé de traces de dogmatisme. En particulier, j'ai toujours gardé en mémoire une simple note de bas de page, lumineuse, où les auteurs expliquent que le concept de "pouvoir " est logiquement impensable dans le cadre de paradigmes déterministes, puisque le pouvoir n'est rien d'autre que la capacité de faire "advenir" des situations, ce qui suppose forcément que ces situations n'étaient pas inéluctables. A quoi me fallait-il renoncer: au déterminisme historique ou au concept de pouvoir? Le choix était facile à faire et la lecture de ce texte m'avait fourni un argument imparable contre toutes les sociologies radicalement déterministes. Bien plus tard, j'ai été amené dans le cadre d'une formation, à écrire quelques notes pédagogiques sur le concept de "pouvoir »C'est à cette occasion seulement que j'ai lu le percutant petit essai de Steven Lukes : "Power. A radical view " qui, bien que très proche du marxisme analytique, avait été remarqué par Crozier et Friedberg dans leur approche du pouvoir. En reprenant et retravaillant ces quelques réflexions, directement inspirées par Lukes (mais qui ne lui sont pas forcément fidèles pour autant) je m'acquitte ainsi d'une petite partie de la dette intellectuelle à l'égard de mon Professeur, sans rien lui concéder sur le plan théorique, bien entendu : les macro-régulations, cela existe ${ }^{1}$.

Proposition 1 (définition) : le pouvoir, c'est la capacité que l'on a de faire faire à quelqu'un quelque chose qu'il ne ferait pas en dehors de cette capacité (ou de l'empêcher de faire quelque chose qu'il ferait en dehors de cette capacité).

Prenons tout de suite un exemple : si mon fils a envie de fumer un joint, que je lui explique longuement pourquoi je ne veux pas et qu'il s'en abstient parce que je le lui ai interdit, alors j'ai exercé un pouvoir sur lui.

On pourrait me répondre qu'il ne s'agit pas de pouvoir mais «d'autorité morale» ou de « force de conviction » ou « capacité d'argumenter ». Il n'en reste pas moins que j'ai, par mon intervention, modifié la conduite que mon fils aurait eue et que, selon la définition classique, j'ai bien exercé sur lui un pouvoir. La nature des ressources utilisées pour exercer ce pouvoir est une autre question, qui a son importance bien sûr. La définition classique de la notion de pouvoir est donc très large et elle embrasse une multitude de formes différentes de pouvoir. Mais malgré cette définition très large, on verra que les utilisations courantes du mot « pouvoir » ne la respectent pas toutes et qu'on utilise parfois le mot dans des circonstances où il ne paraît pas très approprié.

Lorsqu'on dit, par exemple, d'une bombe qu'elle a « le pouvoir de tout raser dans un rayon de cinq cents mètres », s'agit-il de pouvoir? Ou bien, lorsqu'on dit d'une molécule

\footnotetext{
${ }^{1}$ Il s'ensuit que le présent texte n'a aucunement la prétention de constituer un article scientifique, plutôt une invitation à la réflexion. En particulier, ma dette intellectuelle ne se limite pas aux auteurs cités mais s'étend à toutes mes lectures sociologiques.
} 
pharmaceutique qu'elle a le « pouvoir d'empêcher certains types de cellules cancéreuses de se reproduire $^{2}$ », parle-t-on réellement de pouvoir ? Ce sont là des usages courants du mot, mais ils sont trompeurs ou, au minimum, métaphoriques. Une bombe n'a pas plus le "pouvoir» d'opérer des destructions qu'un caillou n'a le "pouvoir» de tomber lorsqu'on le lâche. Pourquoi ? Parce que le "pouvoir» suppose toujours, dans le chef de qui le détient, ou le subit, la capacité d'agir autrement. L'idée de pouvoir implique toujours que l'aboutissement d'une situation est au moins partiellement indéterminé. Si, quoi que je fasse, les choses se produisent inéluctablement d'une façon qui était pré-déterminée, je n'ai aucun pouvoir. Or, dans le monde physique, c'est ainsi que cela se passe : les causes produisent des effets, point à la ligne. Dans le monde social, le pouvoir est toujours une capacité de produire un effet. Et qui dit capacité de le produire, dit aussi capacité de ne pas le produire. Autrement dit, l'idée même du pouvoir n'a pas sa place dans une explication qui se ramène exclusivement à une série de causes et d'effets. D'où la proposition suivante.

\section{Proposition 2 : le pouvoir de faire quelque chose implique toujours que ce quelque chose aurait pu ne pas se produire. Il implique donc aussi la possibilité pour le détenteur du pouvoir de ne pas faire la chose en question.}

Cette proposition emporte également une implication pratique, dont on n'est pas toujours conscient: exercer un pouvoir peut se faire aussi bien par l'abstention que par l'action. Supposons, par exemple, un policier qui assiste à une agression. En intervenant pour l'empêcher, il exerce un certain pouvoir. En n'intervenant pas alors qu'il aurait pu, il exerce le même pouvoir. Dans la régulation sociale, beaucoup d'actes d'exercice du pouvoir sont en fait des abstentions : on aurait pu favoriser ou empêcher un événement particulier, mais on ne l'a pas fait. Il y a ainsi, dans toute société, des pouvoirs qui se voient peu, parce qu'ils ne se manifestent pas dans des grandes décisions dramatiques, mais plutôt dans le fait de «fermer les yeux »

\section{Proposition 3 : le pouvoir est très rarement distribuée de manière équilibrée mais il n'est jamais totalement unilatéral.}

On oppose souvent, dans les discours courants, ceux qui ont du pouvoir et ceux qui n'en ont pas. Même si cette description nous est compréhensible, elle est aussi trompeuse : très rares sont les situations où le pouvoir est tout entier d'un seul côté. Prenons un exemple, à nouveau. Si je reçois un étudiant pour un examen, on dira volontiers que je dispose d'un pouvoir sur lui : de ma cote, beaucoup de choses peuvent dépendre. Mais l'étudiant dispose aussi d'un pouvoir sur moi : il peut, s'il ne s'estime pas traité selon ses mérites, tenter d'infléchir mon avis ; il peut, s'il n'obtient pas satisfaction, me faire la réputation d'un «salaud » auprès du reste de la classe ; il peut se plaindre au Conseil des Etudes, et me mettre dans l'obligation de me justifier; il peut influencer l'évaluation pédagogique. Il peut même, le cas échéant, saisir une juridiction administrative et me mettre en difficulté face à l'institution... Et je ne parle pas de menaces plus physiques, qui sont extrêmement rares à l'Université.

Le pouvoir n'est donc jamais «d'un seul côté ». Il ne pourrait l'être que si quelqu'un contrôlait autrui soit par une contrainte physique absolue (j'empêche matériellement quelqu'un de bouger) soit par une contrainte psychologique absolue (je contrôle parfaitement l'esprit de quelqu'un d'autre).

${ }^{2}$ La plupart de mes exemples sont bien entendu inventés, mais je tenterai de ne prendre que des exemples vraisemblables, à défaut de toujours trouver des exemples réels. 
L'exemple de ce qui se passe en Irak aujourd'hui est à cet égard très révélateur. On voit une «hyper-puissance » qui a le plus grand mal à imposer sa volonté à des groupes faibles en nombre, comparativement très mal armés et divisés. Les Etats-Unis sont donc loin d'être « tout puissants » même si le rapport des forces est largement en leur faveur. On est donc devant un pouvoir très déséquilibré, mais qui n'est pas unilatéral. Il paraît très difficile d'imaginer un exemple dans lequel le plus faible ne dispose d'aucune ressource de pouvoir. Prenons cette arme du faible qui est la menace de suicide par exemple. Elle peut intervenir dans une relation affective ("si tu me quittes, je me suiciderai ») mais aussi dans des relations beaucoup plus politiques (la grève de la faim de prisonniers politiques ou de sans papier). Dans ce cas, le pouvoir s'exerce par la menace d'infliger à l'autre soit une culpabilité personnelle considérable, soit l'opprobre devant l'opinion publique. C'est un exercice coûteux, certes, mais il s'agit bien d'un pouvoir : en menaçant d'un suicide, on amène souvent le destinataire de la menace à modifier son comportement. Le plus faible a donc toujours un ultime pouvoir : mettre en jeu sa vie même. On découvre aujourd'hui l'efficacité redoutable de ce pouvoir à travers les attentats-suicides des kamikazes islamistes.

\section{Proposition 4 : Il paraît raisonnable de limiter la notion de pouvoir aux cas de conflits d'intérêt, au moins potentiels ${ }^{3}$.}

Quel est l'avantage de cette idée ? C'est de distinguer le "pouvoir» de la multitude des formes de l'influence sociale. L'influence mutuelle, dans son sens le plus général, est la matière même des relations sociales : nous ne pouvons pas nous empêcher d'influencer les autres et d'être influencés par eux. Exemple tout simple : je viens d'appeler un ascenseur et, lorsque la porte s'ouvre, il y a déjà une personne dedans. La simple présence de cette personne va modifier mon comportement (si j'avais envie de me gratter l'oreille, il est probable que je m'en abstiendrai). Va-t-on dire pour autant que cette personne exerce un " pouvoir » sur moi ? Ou que j'exerce un « pouvoir » sur elle ? On pourrait, mais le concept de pouvoir deviendrait alors tellement vague qu'il ne nous aiderait plus beaucoup à décrire la réalité sociale. Autre exemple : je fais remarquer à quelqu'un que son lacet est défait et qu'il risque de se casser la figure. Quoi qu'il décide de faire, ma remarque l'aura influencé. Mais il ne paraît pas raisonnable de dire que j'ai « du pouvoir » sur lui dans ces circonstances.

L'idée de lier pouvoir et "conflit d'intérêt» (potentiel ou effectif) paraît tout à fait raisonnable, mais elle pose évidemment de nombreuses difficultés : qui est juge de mon intérêt? Si je prends le train sans payer et qu'un contrôleur m'oblige à descendre, il exerce clairement un pouvoir sur moi : mon intérêt personnel (illégitime, certainement, mais ce n'est pas la question), c'est de voyager sans payer. L'intérêt de la $\mathrm{SNCB}^{4}$, c'est au contraire de me faire payer mon billet. Il y a donc un conflit d'intérêts. Mais que se passe-t-il si mon médecin me dit « je vous interdis dorénavant le cigare, dans votre propre intérêt ». Est-ce bien de mon intérêt qu'il s'agit ou plutôt de la manière dont le médecin le voit? Et si je préfère, moi, en toute connaissance de cause, une vie plus risquée mais avec plus de plaisir ?

\footnotetext{
${ }^{3}$ Ce point est directement emprunté à Steven Lukes.

${ }^{4}$ Qui est aussi, soit dit en passant, l'intérêt des voyageurs qui ont payé leur billet : ceux qui ne paient pas voyagent non pas gratuitement, comme ils l'imaginent, mais aux frais des autres. Parce que les frais liés aux « resquilleurs » grèvent le budget de la SNCB d'un montant qui aurait pu être affecté à l'amélioration du service ou à la diminution des tarifs.
} 
Proposition 5: : dans les sociétés contemporaines (et sans doute dans la plupart des sociétés humaines), le pouvoir est principalement la faculté d'obtenir le consentement de ceux sur qui il s'exerce.

Ainsi, dans un état de droit, l'usage de la force n'est pas abandonné mais son exercice est réservé à la puissance publique, c'est-à-dire à l'Etat au sens large et à ses organes. Dès lors, par exemple, l'importance des discussions tout à fait contemporaines sur le rôle des sociétés de gardiennage ou des milices privées. Leur accorder le droit de faire usage de la force (fouiller un client à la sortie d'un magasin) n'est-ce pas revenir à une forme tout à fait discréditée de pouvoir (le pouvoir des milices privées) ? Par contre on contestera moins l'usage par un vigile de son influence, comme de demander assez autoritairement au client d'accepter volontairement d'être fouillé parce qu'on le soupçonne d'avoir caché une marchandise quelconque. On ne lui contestera pas non plus une forme de coercition, qui s'exprimerait par: "Si vous ne vous soumettez pas à une fouille volontaire, nous devrons alors appeler la police ». Dans tous les cas, il s'agit bien de l'exercice d'un pouvoir, mais tous ces modes d'exercice ne nous paraissent pas également légitimes.

Autre débat : la manipulation. Parler de «manipulation » discrédite presque automatiquement l'usage d'un pouvoir, car la manipulation nous paraît toujours illégitime. Dès lors, la tentation est grande d'accuser un argument d'être « manipulateur » simplement parce qu'il déstabilise nos convictions ou que nous n'arrivons pas à y apporter une réponse satisfaisante. Mais cette attitude n'amène-t-elle pas à disqualifier le débat contradictoire, qui est au cœur de toute procédure démocratique? Ainsi, par exemple, on peut comprendre que les opinions publiques occidentales se soient senties «manipulées » par certains de leur dirigeants en découvrant que les fameuses «Armes de Destruction Massive» dont l'Irak aurait été doté restaient définitivement introuvables. Mais lorsque Tony Blair dit que «le monde est mieux sans Saddam Hussein », c'est un argument (que l'on peut évidemment juger insuffisant) et pas une manipulation.

Un argument moral peut-il être un instrument d'exercice du pouvoir ? Oui, puisque à partir d'un argument moral, je peux vous amener à faire quelque chose que vous n'auriez pas fait sans cet argument. Ainsi, si un enseignant voit dans une cour de récréation, un adolescent qui rackette un plus petit que lui, il peut utiliser le fameux argument de Kant: "Si tu trouves normal de racketter un plus petit, tu devrais trouver normal que les adultes te fassent la même chose ». Dans certaines circonstances, cet argument à lui seul peut amener l'interlocuteur à modifier son comportement (indépendamment de l'usage de la force, ou de l'influence liée au statut de l'enseignant). On peut dire qu'il y a un conflit d'intérêts : il n'est pas toujours dans notre intérêt d'adhérer à une morale universalisable. Il est même souvent dans notre intérêt (purement égoïste) de nous en dispenser tout en espérant que les autres, eux, y obéiront. Par exemple, le même argument kantien peut être utilisé par un contrôleur s'adressant à un resquilleur dans un le train (voir plus haut): "Que se passerait-il si tout le monde faisait comme vous? " A quoi un cynique peut répondre : "Mais justement, je compte bien que tout le monde ne fera pas comme moi ". Si le contrôleur, par la simple force de son argument moral, réussit à amener le resquilleur à changer son comportement, il s'agira bien de l'exercice d'un pouvoir. D'un autre côté, quel pouvoir est plus légitime que celui qui repose sur la discussion morale?

On pourrait multiplier les discussions sur la légitimité du pouvoir. Ce qui est important, c'est que, la plupart du temps, le pouvoir a une double face, comme une pièce de monnaie: d'un côté, le pouvoir, de l'autre le consentement de celui à qui il s'adresse. En dehors de la force 
pure et de la manipulation, où le destinataire du pouvoir n'a pas la possibilité de consentir ou non, tout pouvoir repose sur un certain degré de consentement.

Cette notion de consentement est essentielle. Elle n'abolit évidemment pas le pouvoir et elle ne le rend pas nécessairement légitime. Mais elle insiste sur le rôle de celui qui «subit » le pouvoir : sans un minimum de coopération de sa part, le pouvoir, le plus souvent, disparait. Le pouvoir des Rois de France sous l'Ancien Régime a pu paraitre considérable, mais il a perdu toute force au moment de la Révolution française, quand le consentement de la bourgeoisie et d'une grande partie des classes populaires a fait défaut. Plus près de nous, dans les années septante, le pouvoir du Shah d'Iran semblait inébranlable, appuyé d'une part sur ce qui était (réellement, et pas pour rire, comme en Irak) la quatrième armée du monde et, d'autre part sur une extraordinaire police politique ${ }^{5}$. Mais lorsque le consentement populaire s'est effondré, ce pouvoir n'a résisté que quelques semaines et le Shah a finalement dû s'enfuir.

Le consentement, minimalement, peut être le fait qu'on cède à la coercition, qu'on plie devant la menace. Mais, la plupart du temps, c'est beaucoup plus complexe : ceux qui subissent le pouvoir lui reconnaissent au moins un minimum de légitimité et ils ne le remettent pas constamment en cause. Ainsi, le pouvoir d'un chef d'entreprise repose, en définitive, sur la menace de licenciement des travailleurs. Mais les salariés ne sont pas constamment en train de se dire «si je ne fais pas ceci, je serai licencié ». La perspective de la menace ne devient présente à la conscience que dans les moments de crise. En temps normal ils prestent leur journée de travail, avec plus ou moins de réticence ou d'enthousiasme, sans avoir en tête la possibilité d'être licencié. Une partie d'entre eux adhère certainement aux objectifs de l'entreprise : ils sont convaincus qu'ils font un «bon produit» et qu'ils font donc quelque chose d'utile. Dans le pire des cas, ils effectuent leur travail de manière routinière, mais sans ressentir constamment la menace.

A l'exemple qui précède, on voit bien l'ambiguïté du «consentement » : celui-ci n'implique pas que nous nous comporterions de la même façon si subitement, toute contrainte disparaissait. Mais cela veut dire que la contrainte passe «à l'arrière-plan » dans la vie quotidienne, voire même que nous adhérons en partie aux exigences des pouvoirs qui s'exercent sur nous. Si, par exemple, on délivrait les diplômes sans examen, il est probable qu'une partie des étudiants cesserait d'apprendre. Mais la grande majorité des étudiants trouvent normal qu'il y ait des examens et qu'ils doivent s'y soumettre. Ils adhèrent donc globalement à la logique du pouvoir de l'examinateur, en partie par routine, mais aussi, certainement parce qu'ils sont conscients que, sans examen, le diplôme ne vaudrait plus grand chose sur le marché du travail. Si demain, les étudiants décidaient qu'ils se moquent bien de la valeur du diplôme, le pouvoir des examinateurs disparaîtrait du jour au lendemain. C'est d'ailleurs une idée qui a paru séduisante à certains moments : souvenons-nous de Mai $68 \ldots$

De même, pas mal d'automobilistes cesseraient de porter leur ceinture si demain, toutes les sanctions disparaissaient. Mais la plupart des automobilistes sont maintenant favorables à l'obligation de la ceinture. On peut en dire autant de l'alcool au volant : il est manifeste que le durcissement des règles (et donc des menaces de sanction) joue un rôle dans le comportement concret de beaucoup de conducteurs. Il suffit de voir comment les attitudes à propos du

\footnotetext{
${ }^{5}$ Peu avant la révolution «des mollahs » en 1979, presque dix pour cents des citoyens iraniens, tous âges confondus, recevaient de l'argent de la Savak, la célèbre police du Shah. Ce qui veut dire que, statistiquement, il y avait un indicateur dans une famille sur deux !
} 
« dernier pour la route » ont évolué au cours de ces dernières années. Mais la grande majorité des conducteurs sont certainement d'accord avec la répression de l'alcoolisme au volant.

Dire que le pouvoir se construit toujours sur le consentement, au moins partiel, de ceux à qui il s'adresse n'est donc pas dire que les gens sont pleinement d'accord où qu'ils feraient la même chose s'ils avaient eux-mêmes le pouvoir. Cela veut dire que les gens sont partiellement convaincus par les idées de ceux qui exercent le pouvoir. Mais cela peut vouloir dire aussi qu'ils ne voient pas de solution alternative, qu'ils trouvent que cela n'en vaut pas la peine, qu'ils ne sentent pas directement concernés ou qu'ils se sentent isolés dans leur désaccord... ou tout cela à la fois.

Le philosophe italien Tony Negri, qui a pourtant été considéré comme un théoricien de la lutte révolutionnaire armée exprime très bien cette idée que, presque toujours, les «sujets»du pouvoir, participent à son maintien : "Le pouvoir, dit-il, n'est pas quelque chose qui règne sur nos têtes, mais quelque chose que nous faisons " ${ }^{6}$

Le consentement est-il un critère pour apprécier la légitimité du pouvoir ? De ce qui précède, on peut déduire que non : on peut « consentir » à des degrés divers, à des pouvoirs qui ne nous apparaissent pas légitimes. Une autre philosophe italien, Antonio Gramsci, théoricien communiste de l'entre-deux guerres, utilisait d'ailleurs le mot "hégémonie » pour signaler que, dans les démocraties européennes, les dominants règnent autant par la force des idées que par la coercition elle-même.

La légitimité est d'ailleurs une notion d'un autre ordre : c'est un jugement qui dépend irréductiblement des valeurs de celui qui l'exprime. Nous jugeons aujourd'hui que le pouvoir communiste soviétique était illégitime parce qu'ils ne respectait pas les principes de la décision démocratique. Mais du point de vue des communistes eux-mêmes, ce gouvernement était parfaitement légitime : il exerçait la «dictature du prolétariat », phase nécessaire pour produire les conditions du communisme. Ce qui justifiait la dictature du prolétariat, c'était qu'elle était censée aboutir à une société où chacun «contribuerait selon ses possibilités », et « recevrait selon ses besoins ». Bref, la société communiste.

Le consentement est donc une notion empirique : on peut s'en faire une idée au degré de révolte qui règne dans une société, au degré de force ou de manipulation auquel un gouvernement est obligé de recourir pour mener sa politique. La légitimité est une notion normative : un pouvoir est légitime pour nous s'il est constitué conformément à nos valeurs.

\section{Proposition 6 : le pouvoir auquel on consent le plus volontiers est celui qui n'est pas perçu comme tel.}

Nos sociétés sont ainsi irriguées par de multiples pouvoirs, très ténus en apparence mais puissants lorsqu'ils sont accumulés et qui sont exercés par des gens qui n'en ont absolument pas conscience sur des gens dont ils ne sauront sans doute jamais rien. Ces pouvoirs sont ceux qui sont issus du fonctionnement même de la société et ils sont, dans notre chef, un simple pouvoir de consentir. Nous offrons aux multinationales, aux financiers internationaux, aux médias, aux hommes politiques les moyens d'exercer leur pouvoir, non par des actes que nous faisons mais parce que nous remplissons nos fonctions de travailleurs, épargnants, consommateurs dans la société telle qu'elle est organisée.

\footnotetext{
${ }^{6}$ Michael Hardt et Antonio Negri : Empire, 10/18, p. 210
} 
J'ai proposé d'appeler ce pouvoir « invisible » parce qu'il n'est ni perçu ni intentionnel. Il me semble qu'il faut néanmoins le traiter comme un réel pouvoir, en cohérence avec la première proposition suggérée supra, à savoir que le pouvoir est toujours un pouvoir de « faire » ou de « ne pas faire ». Dès lors, lorsque nous laissons faire des choses qui ne pourraient pas se faire sans notre consentement, il me semble que nous exerçons un pouvoir. Le pouvoir invisible (on pourrait aussi l'appeler structurel) a ceci de particulier qu'il ne peut guère être identifié et qu'il ne comporte donc aucune responsabilité. Or, il me semble qu'un des traits des sociétés complexes c'est de faire de plus en plus de place au pouvoir sans responsabilité.

Un exemple frappant est la transformation de l'organisation du travail au cours des trente-cinq dernières années : on passe progressivement ${ }^{7}$ de formes très « disciplinaires » d'organisation $\mathrm{du}$ travail à des formes plus autonomes, ou les producteurs disposent d'une marge de manœuvre beaucoup plus grande, en échange de quoi ils sont mis en concurrence les uns avec les autres. Là où, auparavant, tous les salariés obéissaient à des ordres directs de supérieurs identifiables, ils sont de plus en plus souvent, aujourd'hui, confrontés de manière plus ou moins directe aux « clients » de l'entreprise ${ }^{8}$.

Ce pouvoir structurel, c'est ce que le sociologue allemand Ulrich Beck appelle le pouvoir subpolitique : il ne fait pas l'objet d'une décision collective, il est la résultante de l'interaction de décisions individuelles dans un monde où la régulation se fait de plus en plus par la compétition et de moins en moins par la décision collective. Mais cela ne signifie pas que les acteurs, en particulier les plus puissants, n'aient aucune conscience de ce qui s'exerce à travers le système lui-même.

Ce pouvoir-là n'est doté d'aucune légitimité démocratique puisqu'il ne fait appel ni à la délibération, ni à la représentation, ni à la participation. Mais il constitue de plus en plus la trame de notre vie quotidienne. Ce n'est pas un pouvoir démocratique, mais c'est sans doute, tout bien pesé, le pouvoir le plus important dans nos démocraties. La dernière ruse du pouvoir serait-elle en définitive, comme pour le Diable, de nous convaincre qu'il n'existe pas ?

\section{Marc Jacquemain Institut des Sciences Humaines et Sociales Université de Liège 7, bd du Rectorat - 4000 LIEGE}

\section{Sources :}

Crozier M., Friedberg E. L'acteur et le système. Paris, Le Seuil, Coll « Points », 1977. Hardt M., Negri T. Empire, Paris, 10/18, 2000.

Lukes S. Power. A radical view, London, Palgrave, 2005 [1974]

\footnotetext{
${ }^{7}$ J'insiste sur « progressivement » parce que le passage est loin d'être achevé.

${ }^{8}$ Pour un exemple très drôle et très parlant de pouvoir qui s'efforce délibérément de se rendre invisible, on peut faire référence au film de Lars Von Trier «Le directeur » : le propriétaire d'une petite entreprise danoise invente un «grand patron » absent qui serait le décideur ultime et auquel il impute toutes les décisions très peu sociales qu'il prend lui-même.
} 